\title{
On the effective properties of foams in the framework of the couple stress theory
}

Received: 12 November 2019 / Accepted: 12 March 2020 / Published online: 21 March 2020

(C) The Author(s) 2020

\begin{abstract}
In the framework of the couple stress theory, we discuss the effective elastic properties of a metal open-cell foam. In this theory, we have the couple stress tensor, but the microrotations are fully described by displacements. To this end, we performed calculations for a representative volume element which give the matrices of elastic moduli relating stress and stress tensors with strain and microcurvature tensors.
\end{abstract}

Keywords Foam $\cdot$ Effective properties · Couple stress theory $\cdot$ Anisotropy

\section{Introduction}

Polymer and metal foams have rather perspective properties and widely used in the engineering, see, e.g., [1-5]. From the mechanical point of view, foams may demonstrate quite unusual behavior. It is worth to mention that the re-entrant foams have negative Poisson's ratio [6] and belong to the class of auxetic materials [7]. As an open-cell foam can be treated as a beam lattice where bending deformations of struts may play a dominant role, it is natural to consider it as a material with couple stresses. In fact, foams are treated as an example of Cosserat-type materials. In order to find the micropolar material parameters, the straightforward experiments on foams were performed in [8-13].

Let us note that for generalized continua such as media with couple stresses, the determination of material parameters used in constitutive equations constitute a rather complex and important problem. Even in the case of classic (Cauchy-type) materials, this problem was stated as a crucial one by Truesdell, see [14,15]. In addition to aforementioned experimental results, we mentioned here the various homogenization techniques. In fact, the homogenization of composite materials with high contrast in properties may lead to generalized models of continuum, such as micropolar and micromorphic media, or strain- and stress-gradient elasticity,

Communicated by Andreas Öchsner.

Victor A. Eremeyev acknowledges the support of the Government of the Russian Federation (Contract No. 14.Z50.31.0046).

A. Skrzat $(\varangle)$

Department of Material Forming and Processing, Faculty of Mechanical Engineering and Aeronautics,

Politechnika Rzeszowska im. Ignacego Lukasiewicza, al. Powstanców Warszawy 12, 35-959 Rzeszow, Poland

E-mail: askrzat@prz.edu.pl

\section{A. Eremeyev}

Department of Mechanics of Materials and Structures, Faculty of Civil and Environmental Engineering,

Gdańsk University of Technology, ul. Gabriela Narutowicza 11/12, 80-233 Gdańsk, Poland

E-mail: eremeyev.victor@gmail.com

V. A. Eremeyev

Don State Technical University, Gagarina sq., 1, Rostov-on-Don, Russia 344000 
see, e.g., [16-28], and the references therein. Even in the case of isotropic materials undergoing infinitesimal deformations, the constitutive equations contain a lot of elastic parameters. For example, within the linear micropolar elasticity we have six elastic moduli $[29,30]$, so in addition to the classic Lame moduli we require four micropolar moduli. In the Toupin-Mindlin strain-gradient elasticity [31-33], we get five additional elastic moduli, see also [34-38] for discussion about the representation of a strain energy function and tensors of elastic moduli. A particular class of strain-gradient elasticity consists of the couple stress theory called also Cosserat continuum with constraint rotations [29]. A modified couple stress theory with symmetric stress and couple stress tensor was proposed by [39]. In [39], we have only one additional length-scale material parameter in the isotropic case. Let us note that the strain-gradient elasticity and its various models became a fruitful model for modeling of beam lattice and other architectured materials, see [18-28] and the references therein. In particular, the couple stress theory was developed in [40] as a result of homogenization.

The aim of this paper is to discuss the homogenization technique of foams in the framework of the couple stress theory as discussed in [40]. A real geometry of an alumina foam specimen was obtained using the tomography technique. As an open-cell foam has quite complex microstructure advanced technologies as tomography is required to get real geometry as well as extensive calculations, see, e.g., [40-45]. As a result, we obtain the complete set of anisotropic material parameters which can be used for further analysis of this foam materials.

The paper is organized as follows. In Sect. 2, we recall in brief the governing equations of the couple stress theory. In order to demonstrate the used approach, we consider a benchmark solution for an isotropic material in Sect. 3. Here, we consider deformations of a solid cube for different boundary conditions. The derivation of the effective properties is based on Hill-Mandel lemma, that is on the comparison of the mechanical energies of the non-homogeneous material and the effective media [46,47]. To this end, we propose new set of consistent kinematic boundary conditions. Finally, in Sect. 4 we apply this technique for the real tomography-based structures. Here, we obtain 21 elastic moduli for a given metal foam. As a result, we get the complete set of material parameters for the considered foam in the framework of the couple stress theory.

\section{Constitutive equations of the couple stress theory}

The kinematics of a Cosserat-type medium is based on two kinematical descriptors that are the fields of displacements and microrotations

$$
\mathbf{u}=\mathbf{u}(\mathbf{x}), \quad \phi=\phi(\mathbf{x})
$$

where $\mathbf{x}$ is the position vector of material particles. Unlike Cosserat continuum where $\boldsymbol{\phi}$ is independent on $\mathbf{u}$, in the couple stress theory $\boldsymbol{\phi}$ depends on $\mathbf{u}$ as follows $[29,40]$

$$
\phi=\frac{1}{2} \nabla \times \mathbf{u}
$$

where $\nabla$ is the three-dimensional nabla operator and $\times$ stands for the cross product.

In the couple stress theory, we introduce two symmetric strain measures

$$
\boldsymbol{\varepsilon}=\frac{1}{2}\left(\nabla \mathbf{u}+(\nabla \mathbf{u})^{T}\right), \quad \chi=\frac{1}{2}\left(\nabla \boldsymbol{\phi}+(\nabla \boldsymbol{\phi})^{T}\right),
$$

which are strain and microcurvature tensors, respectively.

For anisotropic media with central symmetry, there is a strain energy density given by the formula

$$
W=\frac{1}{2} \boldsymbol{\varepsilon}: \mathbf{C}: \boldsymbol{\varepsilon}+\frac{1}{2} \chi: \mathbf{D}: \chi,
$$

where $\mathbf{C}$ and $\mathbf{D}$ are the fourth-order tensors of elastic moduli and : denotes the inner product in the space of second-order tensors. For (4), we get the stress-strain relations in the form

$$
\sigma=\mathbf{C}: \varepsilon, \quad \mu=\mathbf{D}: \chi,
$$

where $\boldsymbol{\sigma}$ and $\boldsymbol{\mu}$ are the stress and couple stress tensors, respectively. The form of $\mathbf{C}$ and $\mathbf{D}$ was discussed in $[30,48,49]$ for various material symmetries of micropolar solids. Let us only note that this analysis should be modified as here $\chi$ is a traceless tensor. 


\section{Benchmark solution}

For the determination of effective material properties using the homogenization, the volume of the representative volume element plays a very important role. Another important issue is the type of the loading which can be considering in the form of constant traction, kinematic assumptions (prescribed displacements) or a periodic boundary conditions (PBCs). With the increased size of representative volume element, these three types should lead to the same solution. However, a large size of RVE requires solving large boundary-value problems which is both expensive and time-consuming. In most of the problems, the periodic boundary conditions provide the best convergence in the determination of the effective material data (unless the analyzed structure is periodical).

In the analysis of the high-porosity foams, constant traction may generate locally very large deformations of the foam skeleton which results in plasticity and/or buckling phenomena. Because of this strain localization, this type of BCs is not considered here. Thus, in this research prescribed displacements (kinematic load) are assumed. The general approach to determination of the effective properties of porous media in the framework of the couple stress theory has been recently proposed by Goda et al. [40]. In [40], twenty-one numerical tests were performed in order to determine twenty-one couple-stress material elastic parameters. In what follows, we use the approach of [40] with some modifications related to boundary conditions. In order to discuss the proposed approach in more details, we consider a benchmark test for an isotropic homogeneous material and performed all numerical tests for it.

Following Hill-Mandel lemma, here we compare stored energies of a non-homogeneous material with stored energies of heterogeneous material with effective elastic moduli. For example, assuming that displacements are linear functions of coordinates such as

$$
\mathbf{u}=\boldsymbol{\varepsilon} \cdot \mathbf{x}
$$

where $\boldsymbol{\varepsilon}$ is a constant tensor and dot means the scalar product, or in Cartesian coordinates

$$
u_{i}=\varepsilon_{i j} x_{j}
$$

we get that $\chi=0$ and strain energy $U$ of RVE takes the form

$$
U=\frac{V}{2} C_{i j k l} \varepsilon_{i j} \varepsilon_{k l}
$$

where $V$ is the volume of RVE. So choosing one or two components of $\varepsilon_{i j}$ equal to 1 , we can easily calculate the corresponding elastic moduli. To this end, we have to applied proper boundary conditions on the faces of RVE.

With $\mathbf{C}$ in hands, we can determine $\mathbf{D}$ in a similar way. Again, assuming $\chi_{i j}$ constant we can consider the following field of rotations $\phi$

$$
\phi=\chi \cdot \mathbf{x}
$$

or, in the coordinate form,

$$
\phi_{i}=\chi_{i j} x_{j}
$$

we get the average energy in the form

$$
U=\frac{V}{2} C_{i j k l}\left\langle\varepsilon_{i j} \varepsilon_{k l}\right\rangle+\frac{V}{2} D_{i j k l} \chi_{i j} \chi_{k l},
$$

where $\langle\ldots\rangle$ denotes the averaged (mean) value. Again, assuming simple form of $\chi_{i j}$ we can obtain from calculated energy the value of corresponding $D_{i j k l}$. Let us note that here we cannot use both $\varepsilon_{i j}$ and $\chi_{i j}$ constant as in the case of the couple stress theory constant $\chi$ corresponds to rotations given as linear functions of coordinates. As the rotations relate to the displacements through (2), in this case we have a quadratic dependence for $\mathbf{u}$ and linear one for $\varepsilon$. 
As an example, we consider steel with the following properties: Young modulus $E=2 e 5 \mathrm{MPa}$ and Poisson's ratio $v=0.33$. In Voigt's notation, $\mathbf{C}$ takes the form of 6 by 6 matrix

$$
\mathbf{C}=\left(\begin{array}{cccccc}
K+\frac{4}{3} G & K-\frac{2}{3} G & -\frac{2}{3} G & 0 & 0 & 0 \\
& K+\frac{4}{3} G & -\frac{2}{3} G & 0 & 0 & 0 \\
& & K+\frac{4}{3} G & 0 & 0 & 0 \\
& & & G & 0 & 0 \\
\text { sym } & & & & G & 0 \\
& & & & & G
\end{array}\right),
$$

where $K=\frac{E}{3(1-2 v)}$ and $G=\frac{E}{2(1+v)}$ are the bulk and shear moduli. So we have $K+4 / 3 G=2.9633 e 5$ $\mathrm{MPa}, K-2 / 3 G=1.4595 e 5 \mathrm{MPa}$, and $G=7.5188 e 4 \mathrm{MPa}$. Comparison of given material parameters and obtained ones in numerical computations can prove the reliability of the proposed strain energy approach. In the numerical simulations, the $1 \times 1 \times 1 \mathrm{~mm}$ cube is considered, see Fig. 1 . So the volume of the RVE is $V=1$ $\mathrm{mm}^{3}$. The Cartesian coordinate system is located in the RVE centroid.

Here, we used an idea to compare stored energies of a non-homogeneous material with stored energies of heterogeneous material with effective elastic moduli.

1. For computation of $C_{11}$, we assume an uniaxial tension with a uniform strain $\varepsilon_{x x}=1$. To this end, we assume the following boundary conditions

$$
\begin{array}{llll}
u_{x}=x & \text { on } & n_{x} & \text { faces } \\
u_{y}=0 & \text { on } & n_{y} & \text { faces } \\
u_{z}=0 & \text { on } & n_{z} & \text { faces. }
\end{array}
$$

The corresponding shape is given in Fig. 2. The strain energy from the FEM analysis and the corresponding modulus are

$$
\begin{aligned}
U_{\mathrm{FEM}} & =148165 \mathrm{~mJ}, \\
C_{11} & =2 U_{\mathrm{FEM}} / V=296330 \mathrm{MPa} .
\end{aligned}
$$

The latter value coincides with the value of $K+4 / 3 G$.

2. For computation of $C_{22}$, we assume an uniaxial tension with a uniform strain $\varepsilon_{y y}=1$. Here, we assume the following boundary conditions

$$
\begin{array}{llll}
u_{x}=0 & \text { on } & n_{x} & \text { faces } \\
u_{y}=y & \text { on } & n_{y} & \text { faces, } \\
u_{z}=0 & \text { on } & n_{z} & \text { faces. }
\end{array}
$$

The calculated strain energy and the corresponding modulus are

$$
\begin{aligned}
U_{\mathrm{FEM}} & =148165 \mathrm{~mJ}, \\
C_{22} & =2 U_{\mathrm{FEM}} / V=296330 \mathrm{MPa} .
\end{aligned}
$$

3. Computation of $C_{33}$-uniaxial tension with a uniform strain $\varepsilon_{z z}=1$. Assumed boundary conditions (BCs) are

$$
\begin{array}{llll}
u_{x}=0 & \text { on } & n_{x} & \text { faces } \\
u_{y}=0 & \text { on } & n_{y} & \text { faces } \\
u_{z}=z & \text { on } & n_{z} & \text { faces. }
\end{array}
$$

Here, we get again

$$
\begin{aligned}
U_{\mathrm{FEM}} & =148165 \mathrm{~mJ}, \\
C_{33} & =2 U_{\mathrm{FEM}} / V=296330 \mathrm{MPa} .
\end{aligned}
$$

In the last two cases, moduli coincide again with the value of $K+4 / 3 G$. 
4. Computation of $C_{12}$-biaxial tension with a uniform strains $\varepsilon_{x x}=\varepsilon_{y y}=1$ (Fig. 3). BCs are

$$
\begin{array}{llll}
u_{x}=x & \text { on } & n_{x} & \text { faces } \\
u_{y}=y & \text { on } & n_{y} & \text { faces } \\
u_{z}=0 & \text { on } & n_{z} & \text { faces. }
\end{array}
$$

This results in

$$
\begin{aligned}
U_{\mathrm{FEM}} & =442282 \mathrm{~mJ}, \\
C_{12} & =\left(2 U_{\mathrm{FEM}} / V-C_{11}-C_{22}\right) / 2=145952 \mathrm{MPa},
\end{aligned}
$$

which coincides with the value of $K-2 / 3 G$.

5. Computation of $C_{23}$ - biaxial tension with a uniform strains $\varepsilon_{y y}=\varepsilon_{z z}=1$. BCs are

$$
\begin{array}{llll}
u_{x}=0 & \text { on } n_{x} & \text { faces, } \\
u_{y}=y & \text { on } & n_{y} & \text { faces, } \\
u_{z}=z & \text { on } & n_{z} & \text { faces. }
\end{array}
$$

This results in

$$
\begin{aligned}
U_{\mathrm{FEM}} & =442282 \mathrm{~mJ}, \\
C_{23} & =\left(2 U_{\mathrm{FEM}} / V-C_{22}-C_{33}\right) / 2=145952 \mathrm{MPa},
\end{aligned}
$$

6. Computation of $C_{13}$-biaxial tension with a uniform strains $\varepsilon_{x x}=\varepsilon_{z z}=1$. BCs are

$$
\begin{array}{llll}
u_{x}=x & \text { on } & n_{x} & \text { faces, } \\
u_{y}=0 & \text { on } & n_{y} & \text { faces, } \\
u_{z}=z & \text { on } & n_{z} & \text { faces. }
\end{array}
$$

This results in

$$
\begin{aligned}
U_{\mathrm{FEM}} & =442282 \mathrm{~mJ}, \\
C_{13} & =\left(2 U_{\mathrm{FEM}} / V-C_{11}-C_{33}\right) / 2=145952 \mathrm{MPa},
\end{aligned}
$$

It is seen that $C_{12}=C_{23}=C_{13}=K-2 / 3 G$.

7. Computation of $C_{44}$-shear deformation with $\varepsilon_{x y}=1$ (Fig. 4). BCs are

$$
\begin{aligned}
& u_{x}=y / 2 \text { on } n_{x} \text { and } n_{z} \text { faces, } \\
& u_{y}=x / 2 \text { on } n_{y} \text { and } n_{x} \text { faces, } \\
& u_{z}=0 \text { on } n_{z} \text { faces. }
\end{aligned}
$$

This results in

$$
\begin{aligned}
U_{\mathrm{FEM}} & =37594 \mathrm{~mJ}, \\
C_{44} & =2 U_{\mathrm{FEM}} / V=75188 \mathrm{MPa},
\end{aligned}
$$

which confirms $G$ value as it should be. It is worth to note that both prescribed displacements must be applied to all $n_{x}$ and $n_{y}$ faces in order to extort the pure shear state. If $u_{x}$ is applied only on $n_{y}$ faces and $u_{y}$ is applied only of $n_{x}$ faces as in [40] the distorted shape of RVE seems to be correct, but locally near the corners the tension and compression stresses arise and the stress state is far from being the pure shear.

8. Computation of $C_{55}$ - shear deformation with $\varepsilon_{y z}=1$. BCs are

$$
\begin{aligned}
& u_{x}=0 \text { on } n_{x} \text { faces, } \\
& u_{y}=z / 2 \text { on } n_{y} \text { and } n_{z} \text { faces, } \\
& u_{z}=y / 2 \text { on } n_{z} \text { and } n_{x} \text { faces. }
\end{aligned}
$$

This results in

$$
\begin{aligned}
U_{\mathrm{FEM}} & =37594 \mathrm{~mJ}, \\
C_{55} & =2 U_{\mathrm{FEM}} / V=75188 \mathrm{MPa} .
\end{aligned}
$$




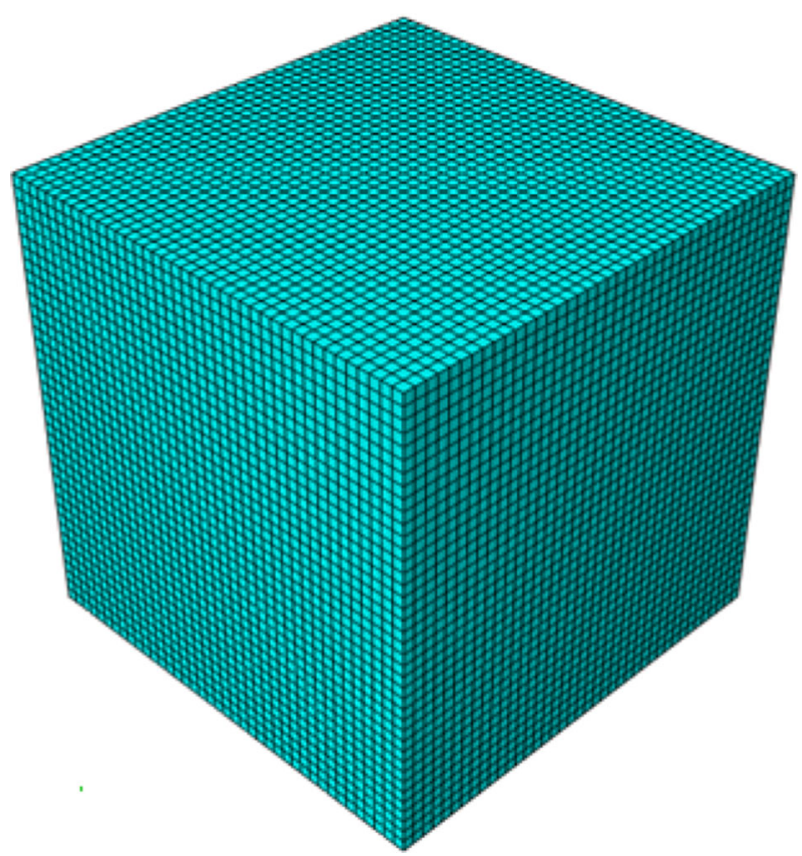

Fig. 1 RVE in the benchmark test
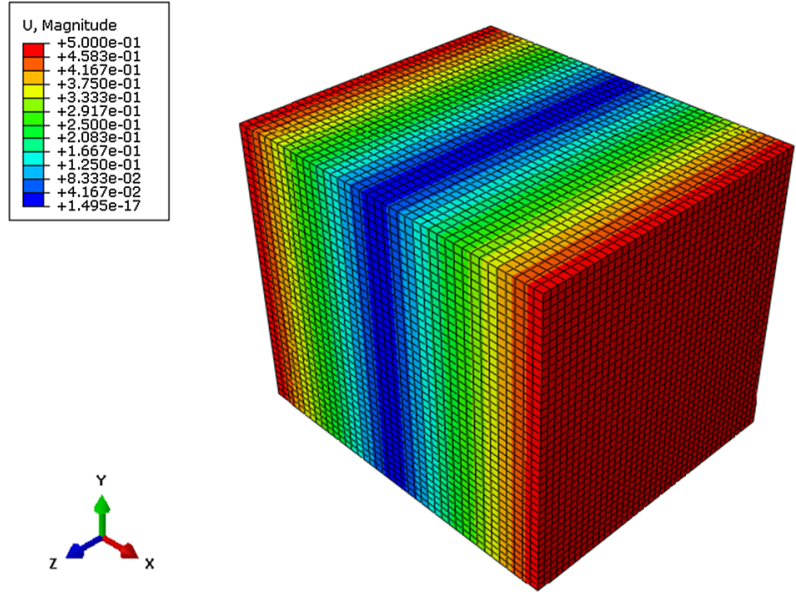

Fig. 2 Displacements of RVE in the prediction of $C_{11}$ parameter

9. Computation of $C_{66}$-shear deformation with $\varepsilon_{x z}=1$. BCs are

$$
\begin{aligned}
& u_{x}=z / 2 \text { on } n_{x} \text { and } n_{z} \text { faces, } \\
& u_{y}=0 \text { on } n_{y} \text { faces, } \\
& u_{z}=x / 2 \text { on } n_{z} \text { and } n_{x} \text { faces. }
\end{aligned}
$$

This results in

$$
\begin{aligned}
U_{\mathrm{FEM}} & =37594 \mathrm{~mJ}, \\
C_{66} & =2 U_{\mathrm{FEM}} / V=75188 \mathrm{MPa} .
\end{aligned}
$$

Now we calculate $D_{i j}$. Here, we also use Voigt's notation as described in [40].

10. For computation of $D_{11}$, we consider an uniaxial torsional rotations with $\chi_{x x}=1$. For such value of $\chi_{x x}$, we get the rotation component $\phi_{x}=x$ and as

$$
\phi_{x}=\frac{1}{2}\left(u_{z, y}-u_{y, z}\right)
$$



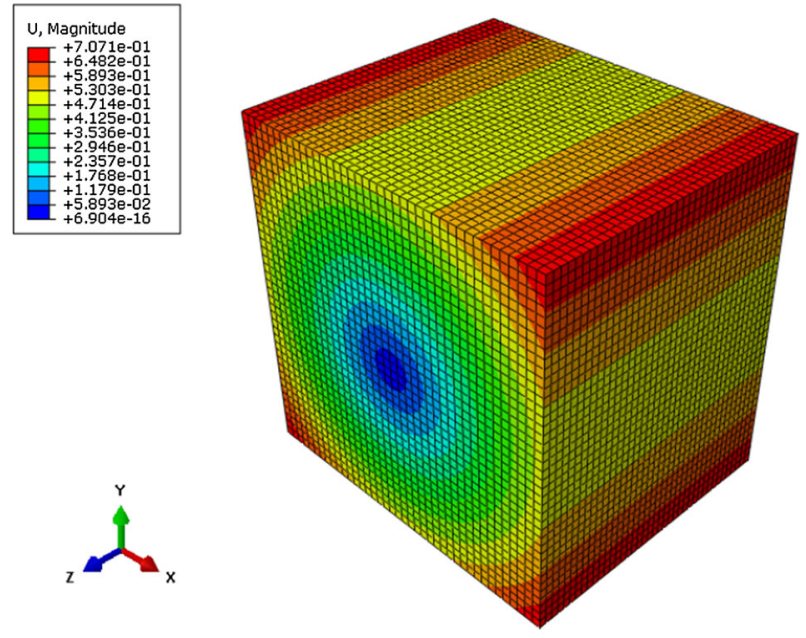

Fig. 3 Displacements of RVE in the prediction of $C_{12}$ parameter
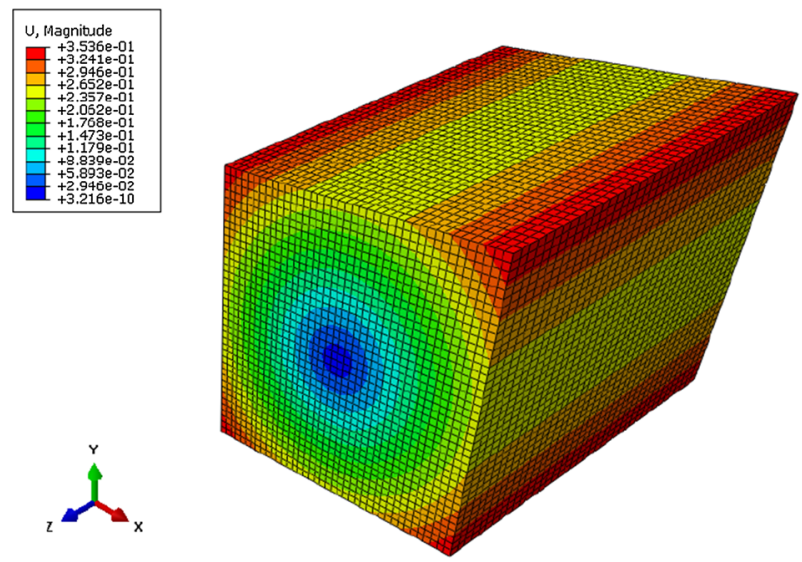

Fig. 4 Displacements of RVE in the prediction of $C_{44}$ parameter

the correspondent displacements are $u_{y}=-x z$ and $u_{z}=x y$. The boundary conditions are

$$
\begin{aligned}
& u_{y}=-x z \text { on } n_{x} \text { faces, } \\
& u_{z}=x y \text { on } n_{x} \text { faces. }
\end{aligned}
$$

The corresponding shape of the RVE is shown in Fig. 5. This results in

$$
\begin{aligned}
U_{\mathrm{FEM}} & =5287.77 \mathrm{~mJ}, \\
D_{11} & =10577.5 \mathrm{~N} .
\end{aligned}
$$

In this case, optionally we can also apply $u_{x}=0$ on $n_{x}$ faces assuming the bounded torsion (without warping). This can be confirmed in experiments made on cylindrical samples. Such boundary conditions can be accepted for the solid cubic samples, but for the porous samples they provide unrealistic deformations. The assumption about bounded torsion overestimates $D_{11}, D_{22}$ and $D_{33}$ parameters, and in consequence $D_{12}, D_{23}$ and $D_{13}$ parameters can became negative. For this reason, we have assumed unbounded torsion (with warping) in the computations of $D_{11}, D_{22}$ and $D_{33}$ parameters.

11. Computation of $D_{22}$-uniaxial torsional rotations with $\chi_{y y}=1$. Here

$$
\phi_{y}=y=\frac{1}{2}\left(u_{x, z}-u_{z, x}\right), \quad u_{x}=y z, \quad u_{z}=-x y .
$$




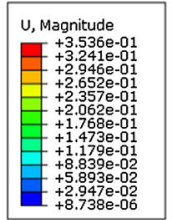

Fig. 5 Displacements of RVE in the prediction of $D_{11}$ parameter
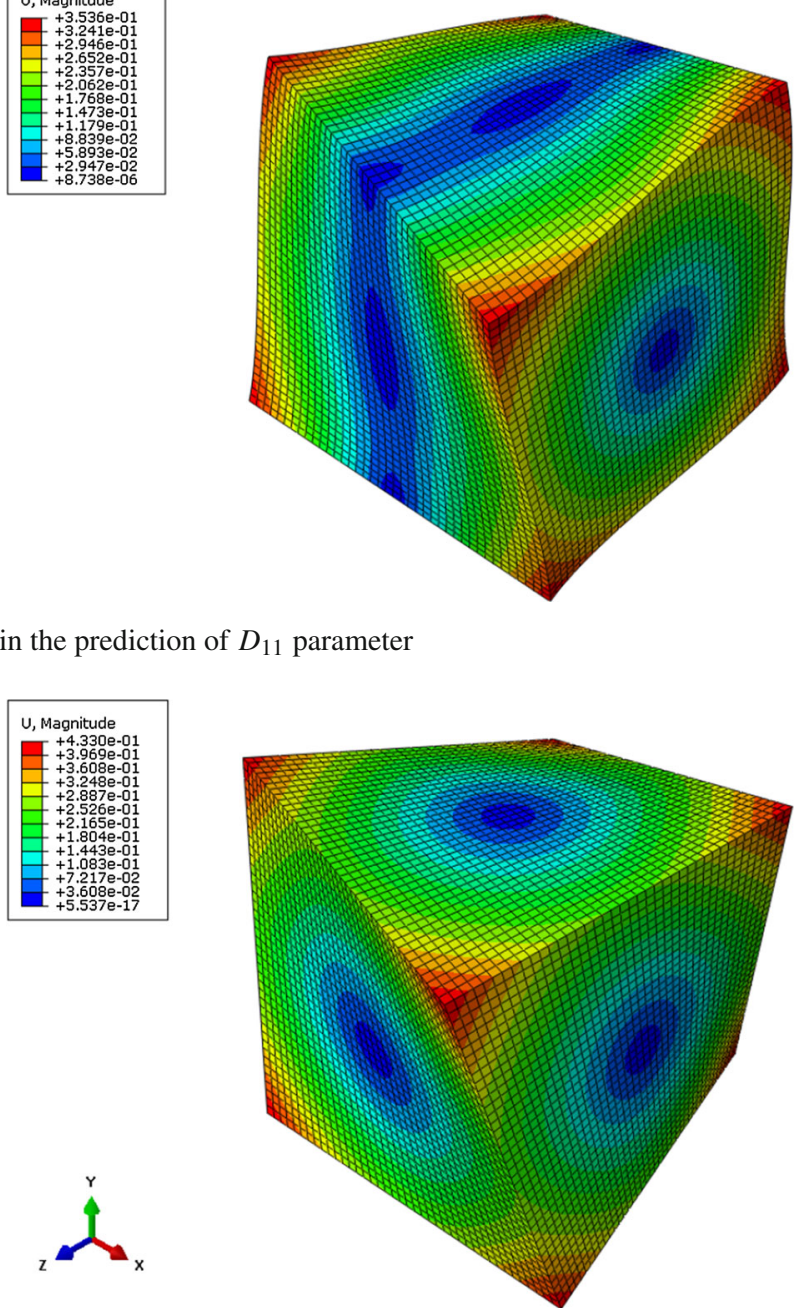

Fig. 6 Displacements of RVE in the prediction of $D_{12}$ parameter

BCs are

$$
\begin{aligned}
& u_{x}=y z \text { on } n_{y} \text { faces, } \\
& u_{z}=-x y \text { on } n_{y} \text { faces. }
\end{aligned}
$$

This results in

$$
\begin{aligned}
U_{\mathrm{FEM}} & =5287.77 \mathrm{~mJ}, \\
D_{22} & =10577.5 \mathrm{~N} .
\end{aligned}
$$

Note that here we have the same value as for $D_{11}$.

12. Computation of $D_{33}$ - uniaxial torsional rotations with $\chi_{z z}=1$. Here

$$
\phi_{z}=z=\frac{1}{2}\left(u_{y, x}-u_{x, y}\right), \quad u_{y}=x z, \quad u_{x}=-z y .
$$

BCs are

$$
\begin{aligned}
& u_{x}=-y z \text { on } n_{z} \text { faces, } \\
& u_{y}=z x \text { on } n_{z} \text { faces. }
\end{aligned}
$$


This results in

$$
\begin{aligned}
U_{\mathrm{FEM}} & =5287.77 \mathrm{~mJ}, \\
D_{33} & =10577.5 \mathrm{~N} .
\end{aligned}
$$

Here, we have again the same value as for $D_{11}$ and for $D_{22}$.

13. Computation of $D_{12}$-biaxial torsional rotations with $\chi_{x x}=1$ and $\chi_{y y}=-1$ (Fig. 6). Here,

$$
\begin{aligned}
& \phi_{x}=x=\frac{1}{2}\left(u_{z, y}-u_{y, z}\right), \quad \phi_{y}=-y=-\frac{1}{2}\left(u_{x, z}-u_{z, x}\right), \\
& u_{x}=-y z, \quad u_{z}=x y, \quad u_{y}=-x z .
\end{aligned}
$$

BCs are

$$
\begin{aligned}
& u_{x}=-y z \text { on } n_{y} \text { faces } \\
& u_{y}=-z x \text { on } n_{x} \text { faces } \\
& u_{z}=x y \text { on } n_{x} \text { faces } \\
& u_{z}=x y \text { on } n_{y} \text { faces }
\end{aligned}
$$

This results in

$$
\begin{aligned}
U_{\mathrm{FEM}} & =12531.3 \mathrm{~mJ}, \\
D_{12} & =1593.14 \mathrm{~N} .
\end{aligned}
$$

It is worth to note that one of the microrotation gradients (or equivalent torsional moments) should be negative. Otherwise, on the common edges between $n_{x}$ and $n_{y}$ faces the prescribed displacements act in the opposite directions which relates to a certain kinematic inconsistency.

14. Computation of $D_{23}$-biaxial torsional rotations with $\chi_{y y}=1$ and $\chi_{z z}=-1$. Here,

$$
\begin{aligned}
& \phi_{z}=-z=-\frac{1}{2}\left(u_{y, x}-u_{x, y}\right), \quad \phi_{y}=y=\frac{1}{2}\left(u_{x, z}-u_{z, x}\right), \\
& u_{x}=z y, \quad u_{y}=-x z, \quad u_{z}=-x y .
\end{aligned}
$$

BCs are

$$
\begin{aligned}
& u_{x}=y z \text { on } n_{y} \text { faces, } \\
& u_{z}=-y x \text { on } n_{y} \text { faces, } \\
& u_{x}=z y \text { on } n_{z} \text { faces } \\
& u_{y}=-x z \text { on } n_{z} \text { faces. }
\end{aligned}
$$

This results in

$$
\begin{aligned}
U_{\mathrm{FEM}} & =12531.3 \mathrm{~mJ}, \\
D_{23} & =1593.14 \mathrm{~N} .
\end{aligned}
$$

15. Computation of $D_{13}$-biaxial torsional rotations with $\chi_{x x}=1$ and $\chi_{z z}=-1$. Here,

$$
\begin{aligned}
& \phi_{x}=x=\frac{1}{2}\left(u_{z, y}-u_{z, z}\right), \quad \phi_{z}=-z=-\frac{1}{2}\left(u_{y, x}-u_{x, y}\right), \\
& u_{x}=z y, \quad u_{y}=-x z, \quad u_{z}=x y .
\end{aligned}
$$

BCs are

$$
\begin{aligned}
& u_{x}=y z \text { on } n_{z} \text { faces, } \\
& u_{y}=-z x \text { on } n_{x} \text { faces, } \\
& u_{z}=x y \text { on } n_{x} \text { faces, } \\
& u_{y}=-x z \text { on } n_{z} \text { faces. }
\end{aligned}
$$




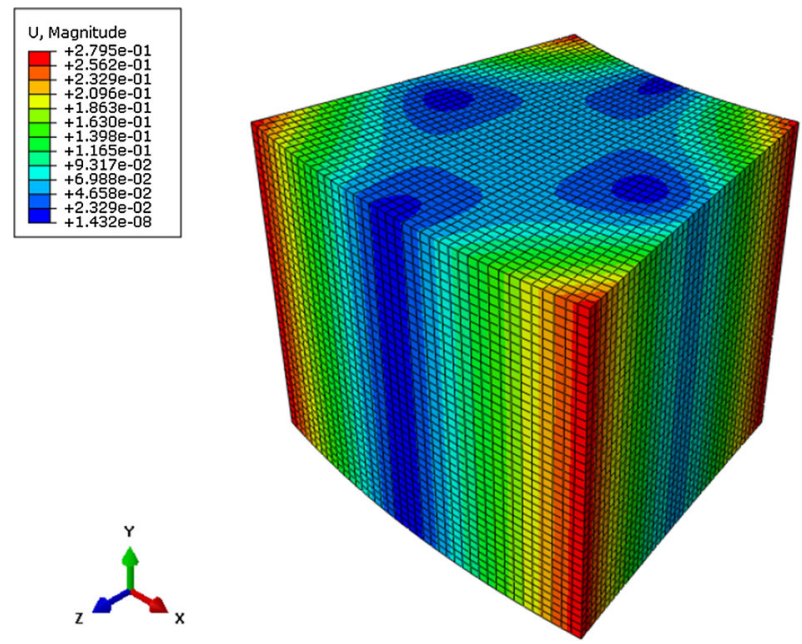

Fig. 7 Displacements of RVE in the prediction of $D_{44}$ parameter

This results in

$$
\begin{aligned}
U_{\mathrm{FEM}} & =12531.3 \mathrm{~mJ}, \\
D_{13} & =1593.14 \mathrm{~N} .
\end{aligned}
$$

16. Computation of $D_{44}$ - uniform curvature $\chi_{x y}=1$ (Fig. 7). Here,

$$
\phi_{y}=x=\frac{1}{2}\left(u_{x, z}-u_{z, x}\right), \quad u_{x}=x z, \quad u_{z}=-\frac{x^{2}}{2} \text {. }
$$

BCs are

$$
\begin{aligned}
& u_{x}=x z \text { on } n_{x} \text { faces, } \\
& u_{y}=0 \text { on } n_{y} \text { faces (optionally), } \\
& u_{z}=-\frac{x^{2}}{2} \text { on } n_{z} \text { faces. }
\end{aligned}
$$

Optionally we can also apply $u_{y}=0$ on $n_{y}$ faces (Fig. 7). This assumption will increase the magnitude of $D_{44}$ parameter about $10 \%$ (in this benchmark test). However, in the case of foams it generates unrealistic solution without warping. This results in

$$
\begin{aligned}
U_{\mathrm{FEM}} & =9350.46 \mathrm{~mJ}, \\
D_{44} & =118700.92 \mathrm{~N} .
\end{aligned}
$$

Without BC $u_{y}=0$ we get

$$
\begin{aligned}
U_{\mathrm{FEM}} & =8425.02 \mathrm{~mJ}, \\
D_{44} & =16850.04 \mathrm{~N} .
\end{aligned}
$$

For the remaining $D_{55}, D_{66}, D_{77}, D_{88}$ and $D_{99}$, we get the same value as for $D_{44}$. Thus, in the following text only the BCs are presented.

17. Computation of $D_{55}$ - uniform curvature $\chi_{y x}=1$. Here,

$$
\phi_{x}=y=\frac{1}{2}\left(u_{z, y}-u_{y, z}\right), \quad u_{y}=-y z, \quad u_{z}=\frac{y^{2}}{2} \text {. }
$$

BCs are

$$
\begin{aligned}
& u_{x}=0 \text { on } n_{x} \text { faces (optionally) } \\
& u_{y}=-y z \text { on } n_{y} \text { faces, } \\
& u_{z}=\frac{y^{2}}{2} \text { on } n_{z} \text { faces. }
\end{aligned}
$$


18. Computation of $D_{66}$-uniform curvature $\chi_{y z}=1$. Here,

$$
\phi_{z}=y=\frac{1}{2}\left(u_{y, x}-u_{x, y}\right), \quad u_{y}=x y, \quad u_{x}=-\frac{y^{2}}{2} .
$$

BCs are

$$
\begin{aligned}
& u_{x}=-\frac{y^{2}}{2} \text { on } n_{x} \text { faces, } \\
& u_{y}=x y \text { on } n_{y} \text { faces, } \\
& u_{z}=0 \text { on } n_{z} \text { faces (optionally). }
\end{aligned}
$$

19. Computation of $D_{77}$-uniform curvature $\chi_{z y}=1$. Here,

$$
\phi_{y}=z=\frac{1}{2}\left(u_{x, z}-u_{z, x}\right), \quad u_{x}=\frac{z^{2}}{2}, \quad u_{z}=-z x .
$$

$\mathrm{BCs}$ are

$$
\begin{aligned}
& u_{x}=\frac{z^{2}}{2} \text { on } n_{x} \text { faces, } \\
& u_{y}=0 \text { on } n_{y} \text { faces (optionally), } \\
& u_{z}=-z x \text { on } n_{z} \text { faces. }
\end{aligned}
$$

20. Computation of $D_{88}$ - uniform curvature $\chi_{x z}=1$. Here,

$$
\phi_{z}=x=\frac{1}{2}\left(u_{y, x}-u_{x, y}\right), \quad u_{y}=\frac{x^{2}}{2}, \quad u_{x}=-x y .
$$

BCs are

$$
\begin{aligned}
& u_{x}=-x y \text { on } n_{x} \text { faces, } \\
& u_{y}=\frac{x^{2}}{2} \text { on } n_{y} \text { faces, } \\
& u_{z}=0 \text { on } n_{z} \text { faces (optionally). }
\end{aligned}
$$

21. Computation of $D_{99}$-uniform curvature $\chi_{z x}=1$. Here,

$$
\phi_{x}=z=\frac{1}{2}\left(u_{z, y}-u_{y, z}\right), \quad u_{y}=-\frac{z^{2}}{2}, \quad u_{z}=z y .
$$

BCs are

$$
\begin{aligned}
& u_{x}=0 \text { on } n_{x} \text { faces (optionally), } \\
& u_{y}=-\frac{z^{2}}{2} \text { on } n_{y} \text { faces, } \\
& u_{z}=y z \text { on } n_{z} \text { faces. }
\end{aligned}
$$

Comparing used boundary conditions with used in [40], one can see that we propose changes in BCs for $C_{44}, C_{55}, C_{66}$, and for $D_{12}, D_{23}, D_{13}, D_{11}, D_{22}, \ldots, D_{99}$. 


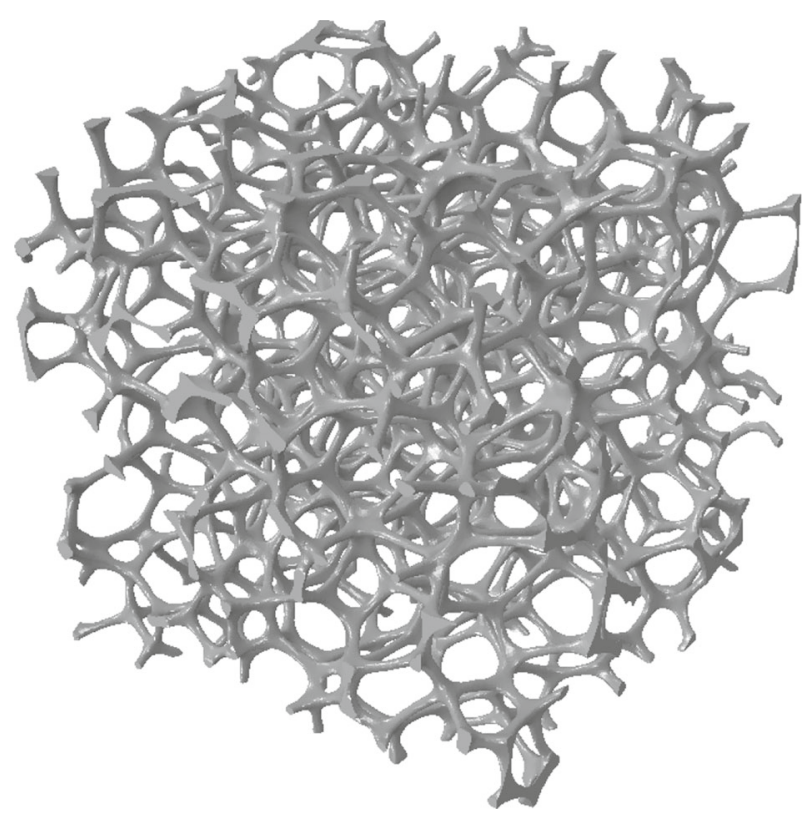

Fig. 8 Geometry of analyzed RVE

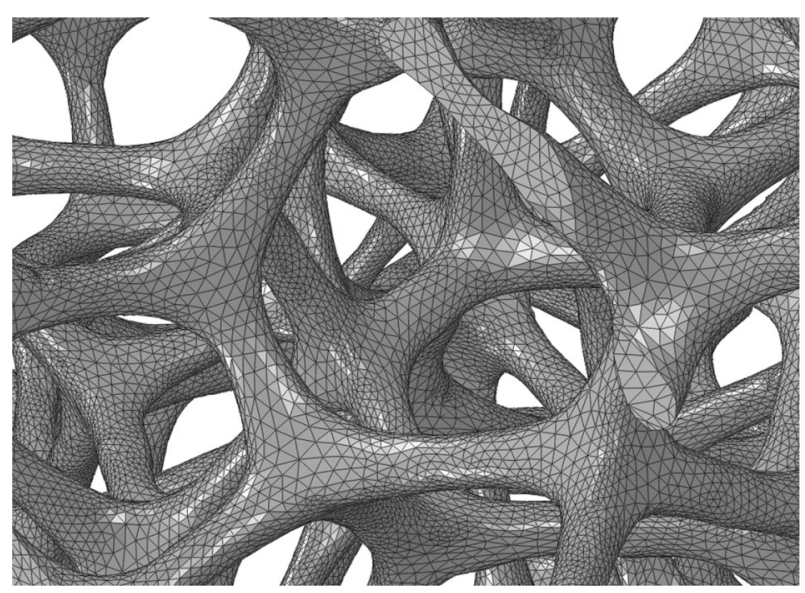

Fig. 9 Enlargement of the FEM mesh

\section{Effective properties for a metal foam}

Following the benchmark test scheme, we perform here the investigations of mechanical properties of the metallic foam. We consider the representative volume element of dimensions $2.5075 \times 2.5075 \times 2.5075 \mathrm{~mm}$ as shown in Fig. 8. The geometrical model of the foam is obtained by microcomputer tomography with the ScanIP+Fe software [45]. The porosity of the foam (the volume of pores related to the RVE volume) is $93.66 \%$. The isotropic material data of the foam are as follows: Young's modulus is $110 \mathrm{GPa}$, Poisson's ratio is 0.34, and the mass density is $8960 \mathrm{~kg} / \mathrm{m} 3$.

The finite element method analysis is made in ABAQUS commercial software. The dense, quite regular mesh consists of tetrahedral elements, see Fig. 9. The size of the problem is large, but reasonable from the point of view of the computation time: the number of nodes is 965,126 , the number of elements is $4,077,000$, and the total number of unknowns is 2,895,606. The computation time of each problem executed on an average PC equipped with $32 \mathrm{~GB}$ memory was about $15 \mathrm{~min}$, which is surprisingly short. It was caused by the effective optimization made by the sparse solver for the analysis of highly porous media. If the solid structure without pores of similar size (about 3 millions of DOFs) is investigated, the execution time would be at least one order longer. 


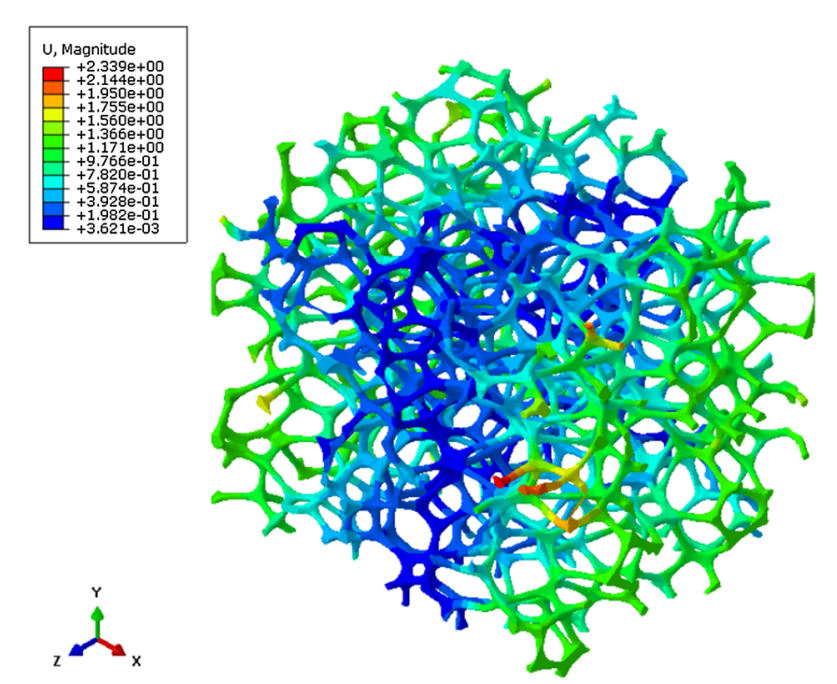

Fig. 10 Displacements of RVE in the prediction of $C_{11}$ parameter

Twenty-one numerical cases necessary for the determination of the couple-stress theory material data are the same as in the benchmark problem. In the computation of $D_{i j}$ parameters, the boundary conditions marked in text as optional are not prescribed. It is caused by the fact that the deformed foam does not preserve the flatness of the not loaded surfaces. Below, we present the foam deformations for all considered kinematic loading cases as well as computed strain energy and the value of the corresponding material parameter. The Cartesian coordinate system is displayed only for visualization of the spatial orientation of the RVE. (The global coordinate system is located in the RVE centroid.) The performed calculations are described in the sequel.

1. Computation of $C_{11}$-uniaxial tension with a uniform strain $\varepsilon_{x x}=1$. The corresponding shape of the RVE is given in Fig. 10. This leads to the following values:

$$
\begin{aligned}
U_{\mathrm{FEM}} & =3219.78 \mathrm{~mJ}, \\
C_{11} & =2 U_{\mathrm{FEM}} / V=408.45 \mathrm{MPa} .
\end{aligned}
$$

2. Computation of $C_{22}$-uniaxial tension with a uniform strain $\varepsilon_{y y}=1$ (Fig. 11). This results in

$$
\begin{aligned}
U_{\mathrm{FEM}} & =3564.07 \mathrm{~mJ}, \\
C_{22} & =2 U_{\mathrm{FEM}} / V=452.12 \mathrm{MPa} .
\end{aligned}
$$

3. Computation of $C_{33}$-uniaxial tension with a uniform strain $\varepsilon_{z z}=1$ (Fig. 12). This results in

$$
\begin{aligned}
U_{\mathrm{FEM}} & =5025.94 \mathrm{~mJ}, \\
C_{33} & =2 U_{\mathrm{FEM}} / V=637.57 \mathrm{MPa} .
\end{aligned}
$$

4. Computation of $C_{12}$-biaxial tension with a uniform strains $\varepsilon_{x x}=\varepsilon_{y y}=1$ (Fig. 13). This results in

$$
\begin{aligned}
U_{\mathrm{FEM}} & =10750.3 \mathrm{~mJ}, \\
C_{12} & =\left(2 U_{\mathrm{FEM}} / V-C_{11}-C_{22}\right) / 2=251.58 \mathrm{MPa} .
\end{aligned}
$$

5. Computation of $C_{23}$-biaxial tension with a uniform strains $\varepsilon_{y y}=\varepsilon_{z z}=1$ (Fig. 14). This results in

$$
\begin{aligned}
U_{\mathrm{FEM}} & =13822.0 \mathrm{~mJ}, \\
C_{23} & =\left(2 U_{\mathrm{FEM}} / V-C_{22}-C_{33}\right) / 2=331.85 \mathrm{MPa} .
\end{aligned}
$$

6. Computation of $C_{13}$-biaxial tension with a uniform strains $\varepsilon_{x x}=\varepsilon_{z z}=1$ (Fig. 15). This results in

$$
\begin{aligned}
U_{\mathrm{FEM}} & =13208.2 \mathrm{~mJ}, \\
C_{13} & =\left(2 U_{\mathrm{FEM}} / V-C_{11}-C_{33}\right) / 2=314.75 \mathrm{MPa} .
\end{aligned}
$$




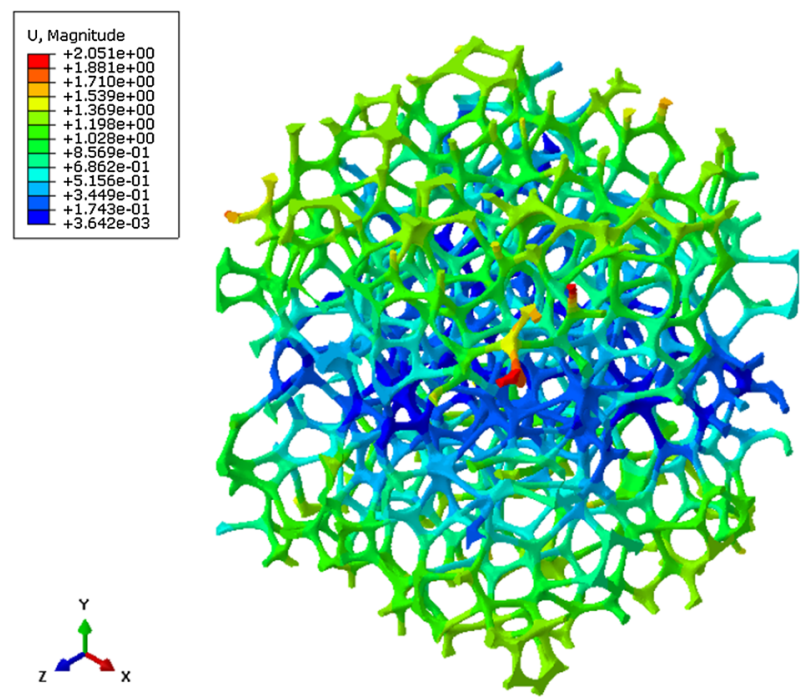

Fig. 11 Displacements of RVE in the prediction of $C_{22}$ parameter

7. Computation of $C_{44}$-shear deformation with $\varepsilon_{x y}=1$ (Fig. 16). This results in

$$
\begin{aligned}
U_{\mathrm{FEM}} & =826.07 \mathrm{~mJ}, \\
C_{44} & =2 U_{\mathrm{FEM}} / V=104.78 \mathrm{MPa} .
\end{aligned}
$$

8. Computation of $C_{55}$-shear deformation with $\varepsilon_{y z}=1$ (Fig. 17). This results in

$$
\begin{aligned}
U_{\mathrm{FEM}} & =738.1 \mathrm{~mJ}, \\
C_{55} & =2 U_{\mathrm{FEM}} / V=93.63 \mathrm{MPa} .
\end{aligned}
$$

9. Computation of $C_{66}$ - shear deformation with $\varepsilon_{x z}=1$ (Fig. 18). This results in

$$
\begin{aligned}
U_{\mathrm{FEM}} & =958.63 \mathrm{~mJ}, \\
C_{66} & =2 U_{\mathrm{FEM}} / V=121.61 \mathrm{MPa} .
\end{aligned}
$$

10. Computation of $D_{11}$ - uniaxial torsional rotations with $\chi_{x x}=1$ (Fig. 19).

This results in

$$
\begin{aligned}
U_{\mathrm{FEM}} & =384.12 \mathrm{~mJ}, \\
D_{11} & =2 U_{\mathrm{FEM}} / V=48.73 \mathrm{~N} .
\end{aligned}
$$

11. Computation of $D_{22}$ - uniaxial torsional rotations with $\chi_{y y}=1$ (Fig. 20). This results in

$$
\begin{aligned}
U_{\mathrm{FEM}} & =439.78 \mathrm{~mJ}, \\
D_{22} & =2 U_{\mathrm{FEM}} / V=55.79 \mathrm{~N} .
\end{aligned}
$$

12. Computation of $D_{33}$ - uniaxial torsional rotations with $\chi_{z z}=1$ (Fig. 21). This results in

$$
\begin{aligned}
U_{\mathrm{FEM}} & =420.69 \mathrm{~mJ}, \\
D_{22} & =2 U_{\mathrm{FEM}} / V=53.37 \mathrm{~N} .
\end{aligned}
$$

13. Computation of $D_{12}$-biaxial torsional rotations with $\chi_{x x}=1$ and $\chi_{y y}=-1$ (Fig. 22). This results in

$$
\begin{aligned}
U_{\mathrm{FEM}} & =914.27 \mathrm{~mJ} \\
D_{22} & =\left(2 U_{\mathrm{FEM}} / V-D_{11}-D_{22}\right) / 2=5.73 \mathrm{~N} .
\end{aligned}
$$




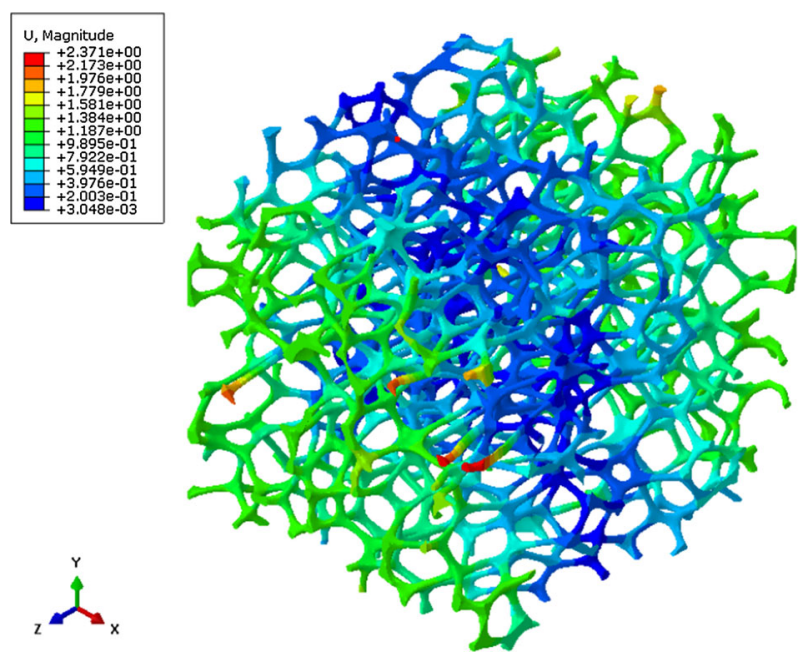

Fig. 12 Displacements of RVE in the prediction of $C_{33}$ parameter
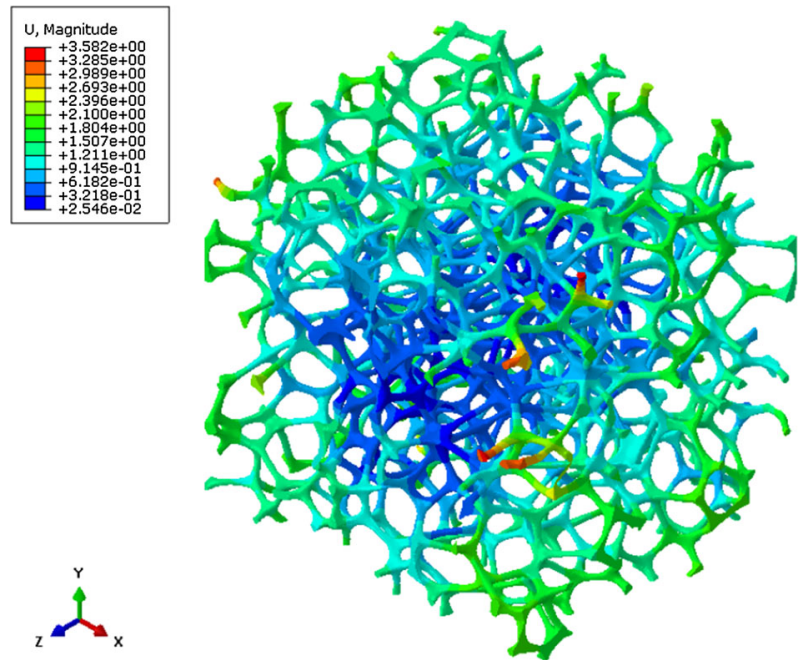

Fig. 13 Displacements of RVE in the prediction of $C_{12}$ parameter

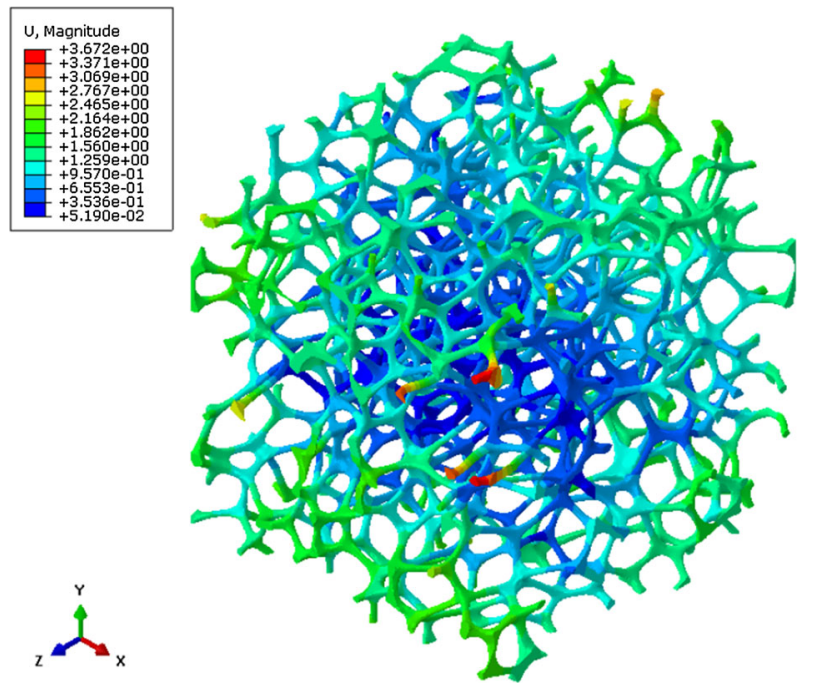

Fig. 14 Displacements of RVE in the prediction of $C_{23}$ parameter 


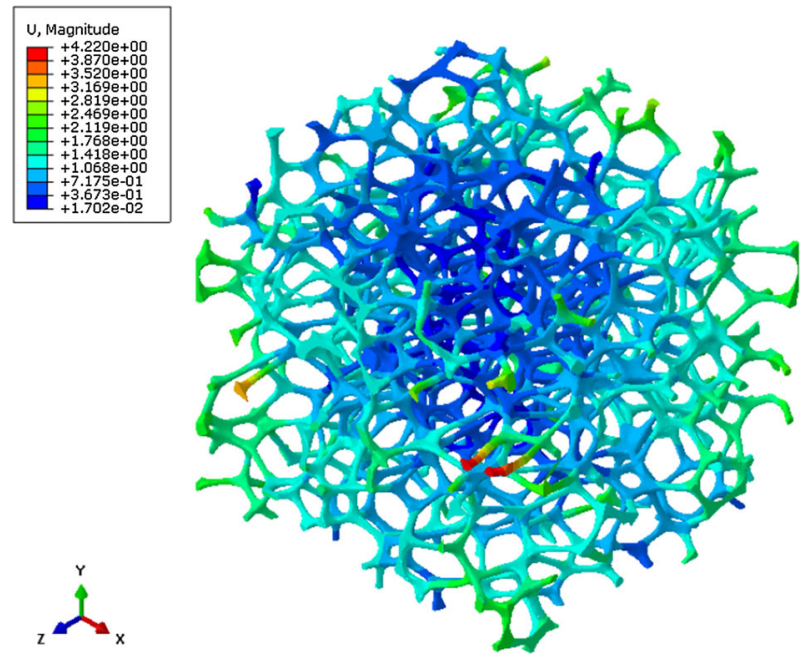

Fig. 15 Displacements of RVE in the prediction of $C_{13}$ parameter

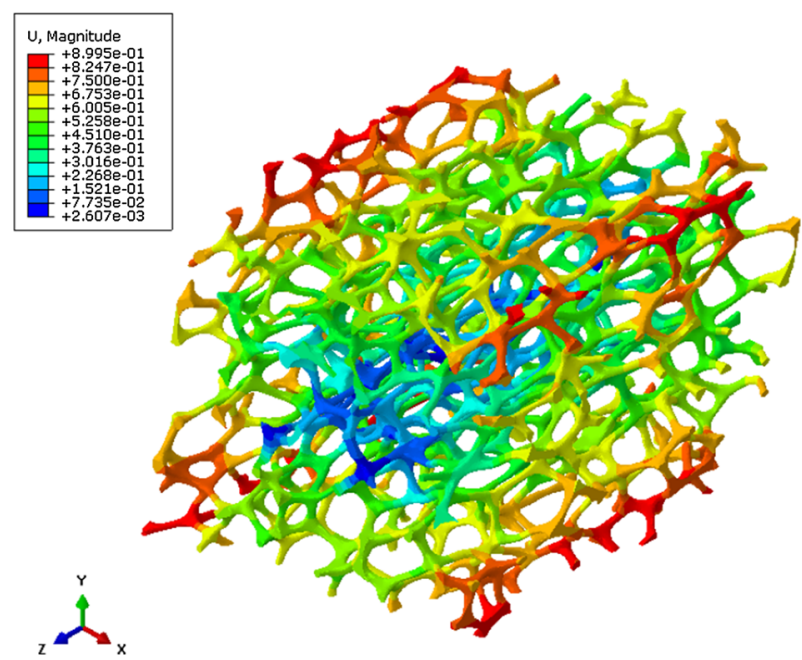

Fig. 16 Displacements of RVE in the prediction of $C_{44}$ parameter

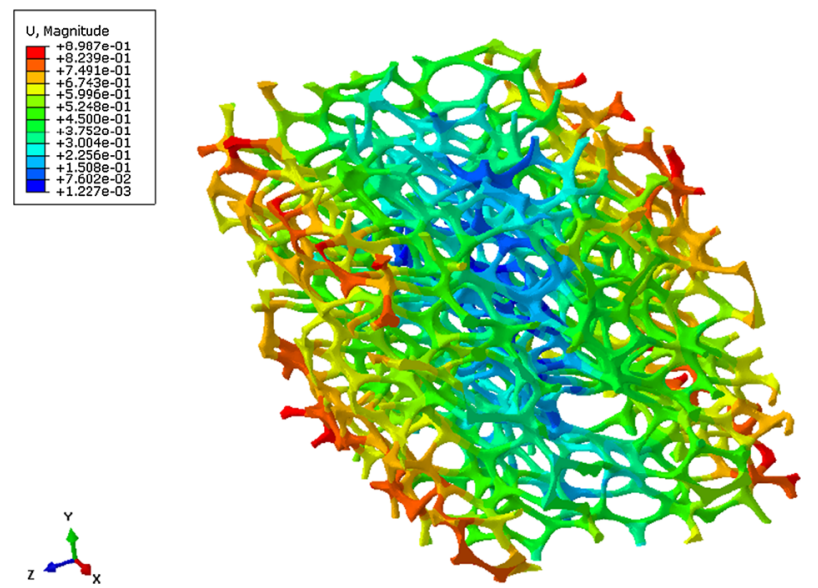

Fig. 17 Displacements of RVE in the prediction of $C_{55}$ parameter 


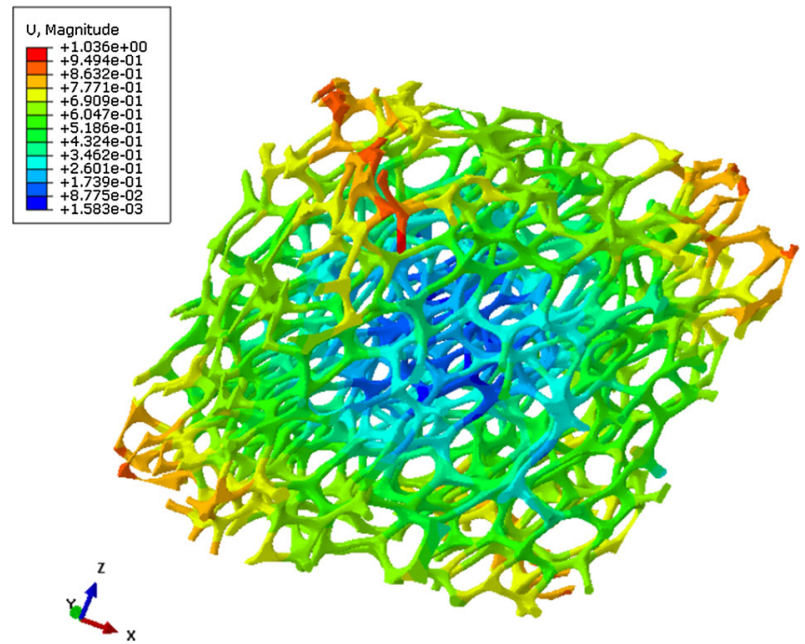

Fig. 18 Displacements of RVE in the prediction of $C_{66}$ parameter
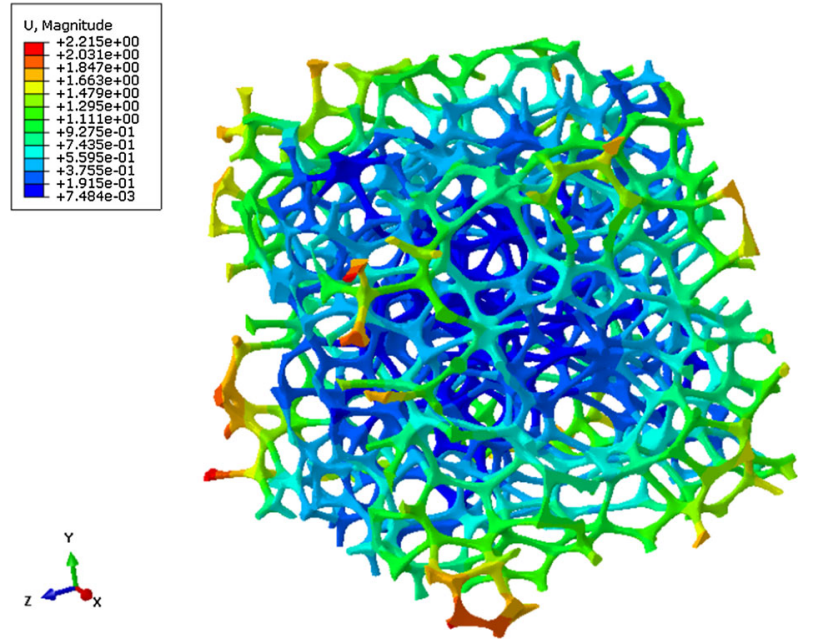

Fig. 19 Displacements of RVE in the prediction of $D_{11}$ parameter

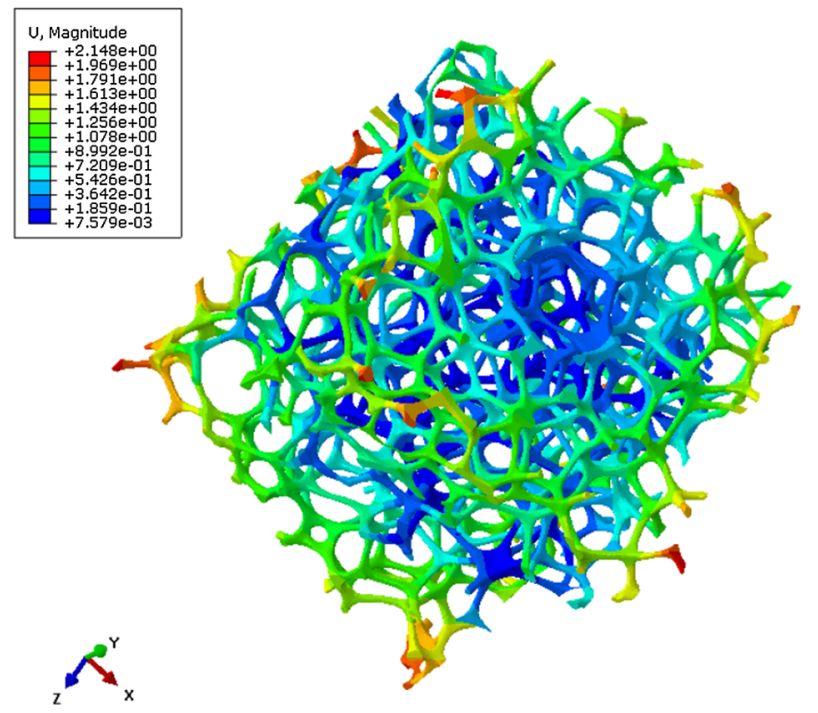

Fig. 20 Displacements of RVE in the prediction of $D_{22}$ parameter 


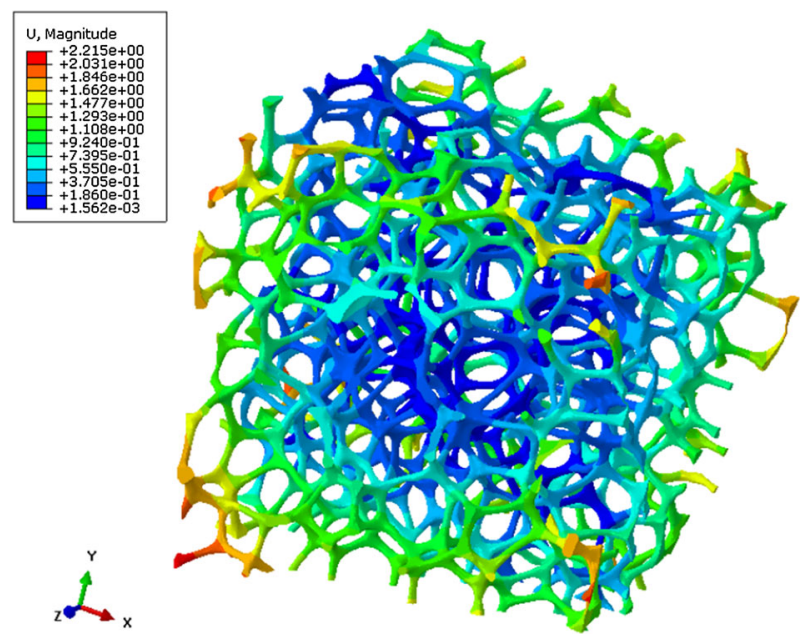

Fig. 21 Displacements of RVE in the prediction of $D_{33}$ parameter
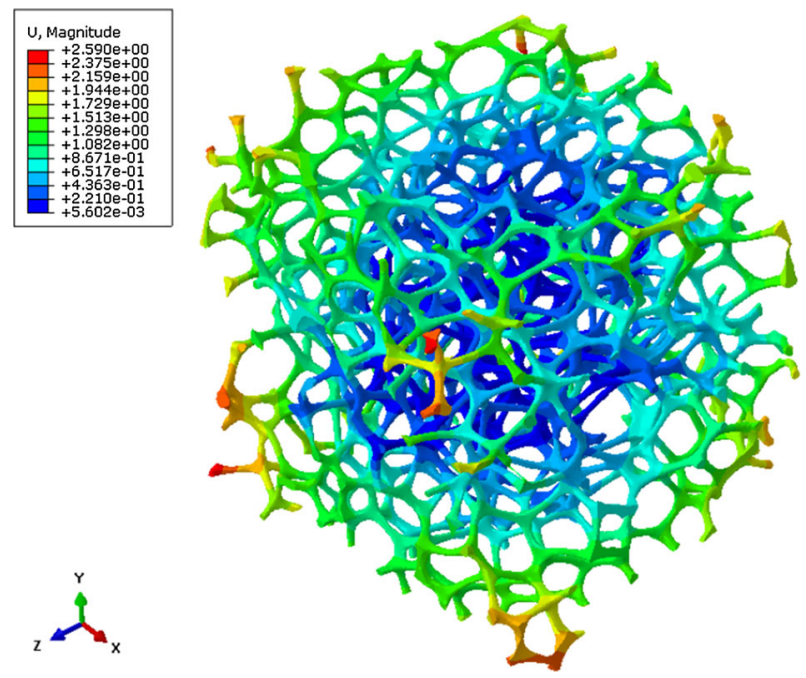

Fig. 22 Displacements of RVE in the prediction of $D_{12}$ parameter
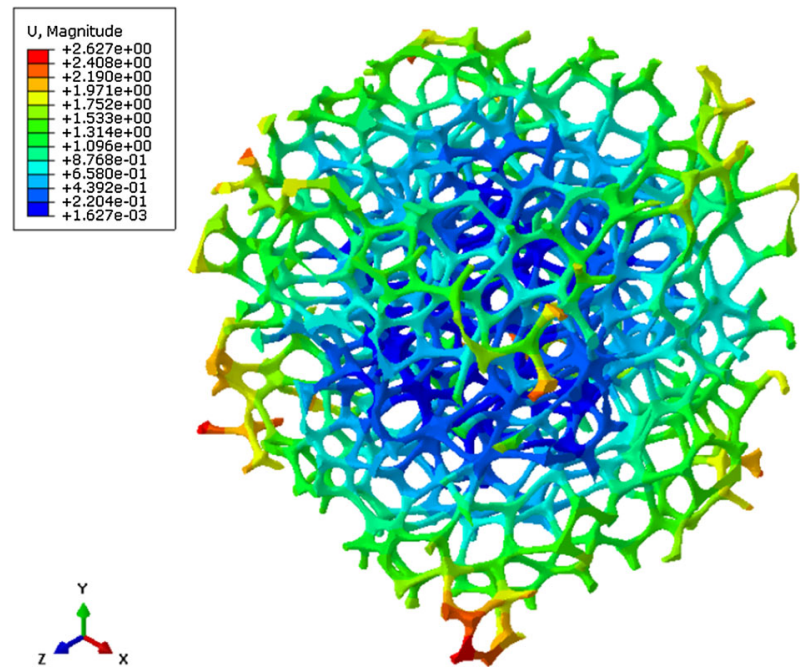

Fig. 23 Displacements of RVE in the prediction of $D_{23}$ parameter 


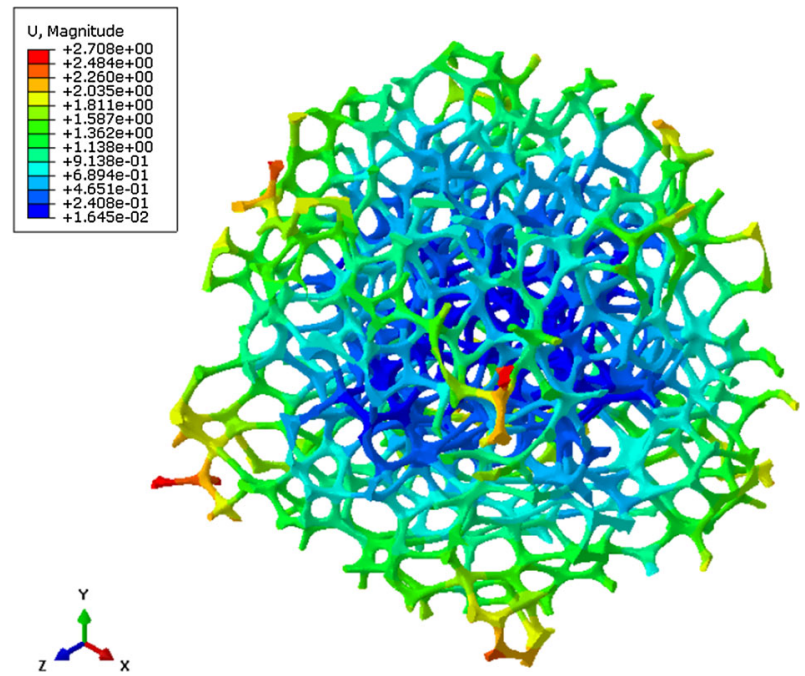

Fig. 24 Displacements of RVE in the prediction of $D_{13}$ parameter

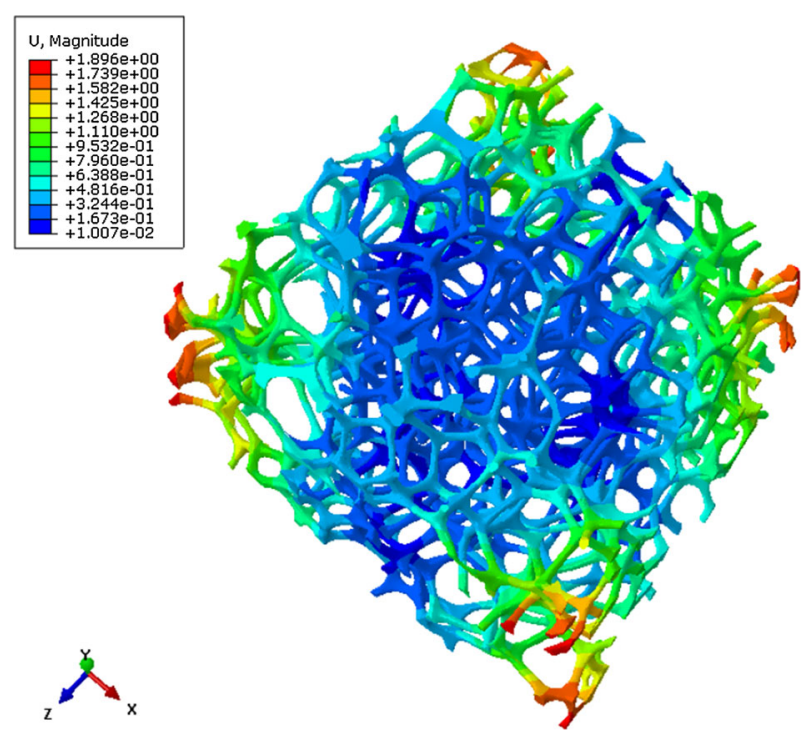

Fig. 25 Displacements of RVE in the prediction of $D_{44}$ parameter

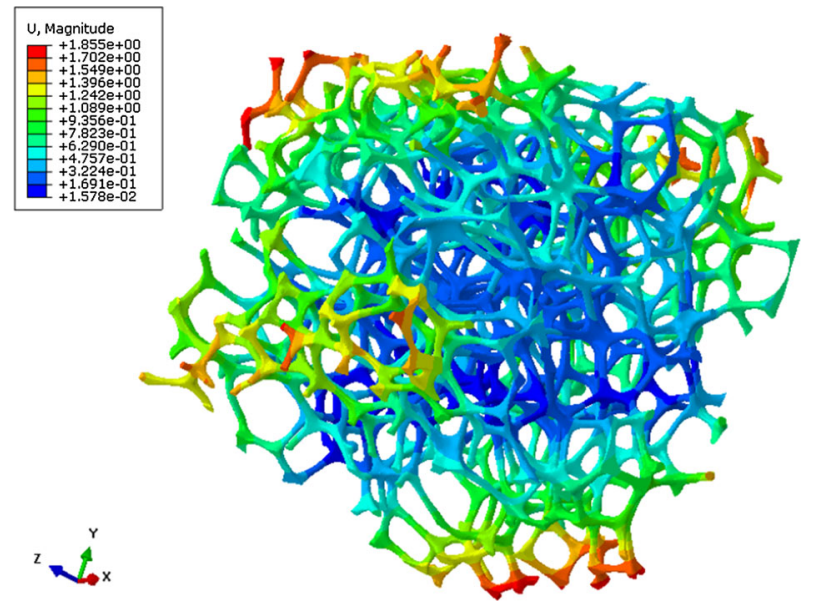

Fig. 26 Displacements of RVE in the prediction of $D_{55}$ parameter 


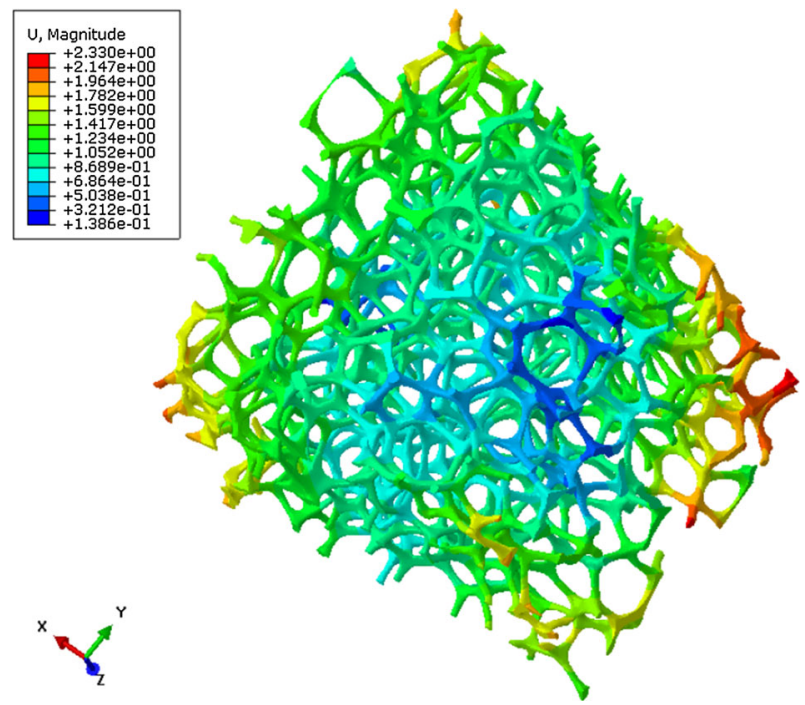

Fig. 27 Displacements of RVE in the prediction of $D_{66}$ parameter

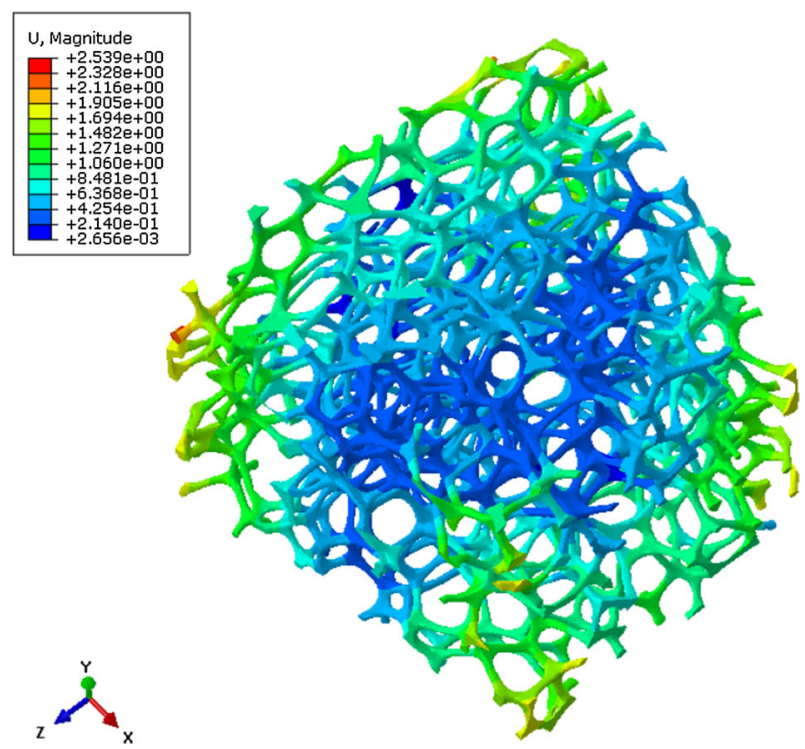

Fig. 28 Displacements of RVE in the prediction of $D_{77}$ parameter

14. Computation of $D_{23}$-biaxial torsional rotations with $\chi_{y y}=1$ and $\chi_{z z}=-1$ (Fig. 23). This results in

$$
\begin{aligned}
U_{\mathrm{FEM}} & =1074.82 \mathrm{~mJ}, \\
D_{22} & =\left(2 U_{\mathrm{FEM}} / V-D_{22}-D_{33}\right) / 2=13.59 \mathrm{~N} .
\end{aligned}
$$

15. Computation of $D_{13}$-biaxial torsional rotations with $\chi_{x x}=1$ and $\chi_{z z}=-1$ (Fig. 24). This results in

$$
\begin{aligned}
U_{\mathrm{FEM}} & =1211.14 \mathrm{~mJ}, \\
D_{13} & =\left(2 U_{\mathrm{FEM}} / V-D_{11}-D_{33}\right) / 2=25.77 \mathrm{~N} .
\end{aligned}
$$

16. Computation of $D_{44}$ - uniaxial torsional rotations with $\chi_{x y}=1$ (Fig. 25). This results in

$$
\begin{aligned}
U_{\text {FEM }} & =836.78 \mathrm{~mJ}, \\
D_{44} & =2 U_{\text {FEM }} / V=106.15 \mathrm{~N} .
\end{aligned}
$$




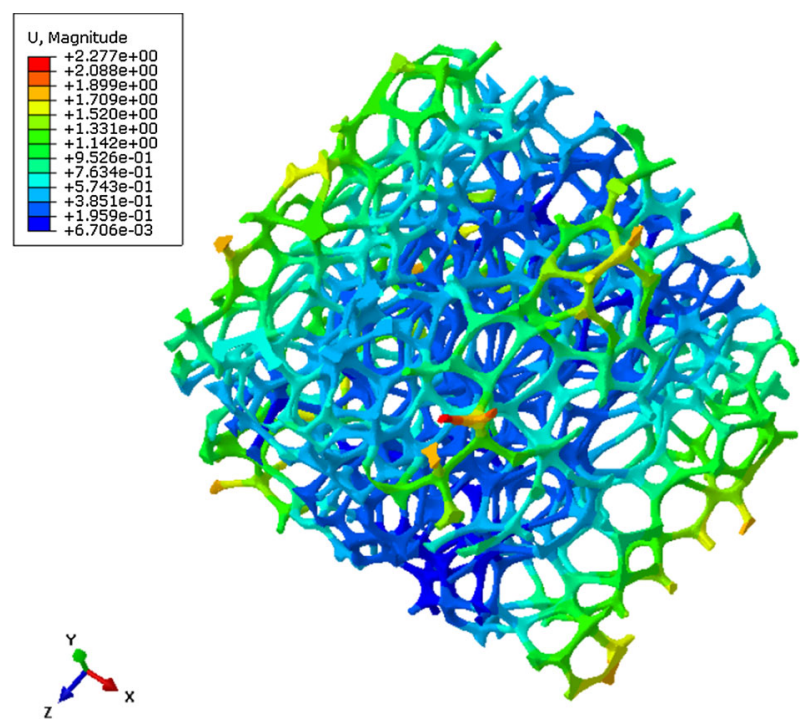

Fig. 29 Displacements of RVE in the prediction of $D_{88}$ parameter

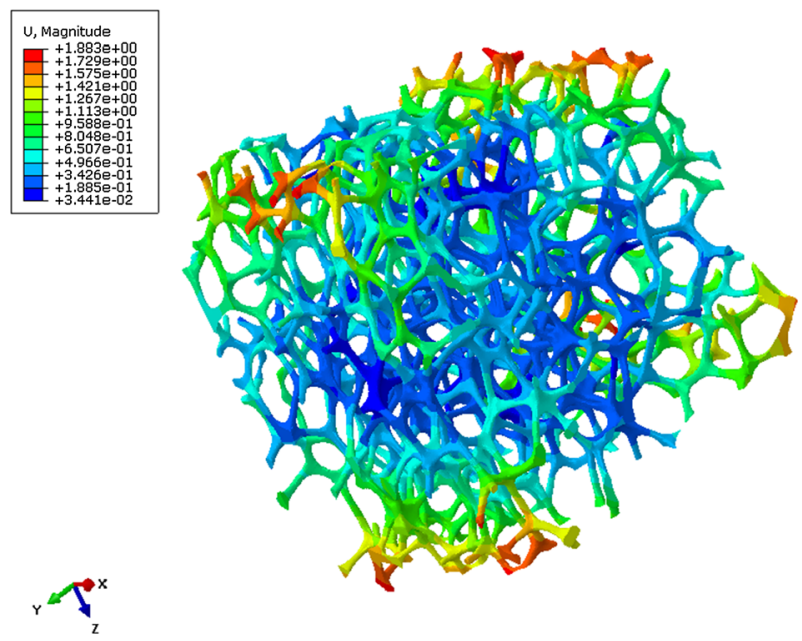

Fig. 30 Displacements of RVE in the prediction of $D_{99}$ parameter

17. Computation of $D_{55}$ - uniaxial torsional rotations with $\chi_{y x}=1$ (Fig. 26). This results in

$$
\begin{aligned}
U_{\mathrm{FEM}} & =911.66 \mathrm{~mJ}, \\
D_{55} & =2 U_{\mathrm{FEM}} / V=115.65 \mathrm{~N} .
\end{aligned}
$$

18. Computation of $D_{66}$ - uniaxial torsional rotations with $\chi_{y z}=1$ (Fig. 27). This results in

$$
\begin{aligned}
U_{\text {FEM }} & =830.71 \mathrm{~mJ}, \\
D_{66} & =2 U_{\text {FEM }} / V=105.38 \mathrm{~N} .
\end{aligned}
$$

19. Computation of $D_{77}$ - uniaxial torsional rotations with $\chi_{z y}=1$ (Fig. 28). This results in

$$
\begin{aligned}
U_{\mathrm{FEM}} & =1134.68 \mathrm{~mJ}, \\
D_{77} & =2 U_{\mathrm{FEM}} / V=143.94 \mathrm{~N} .
\end{aligned}
$$

20. Computation of $D_{88}$ - uniaxial torsional rotations with $\chi_{x z}=1$ (Fig. 29). This results in

$$
\begin{aligned}
U_{\text {FEM }} & =800.25 \mathrm{~mJ}, \\
D_{88} & =2 U_{\text {FEM }} / V=101.52 \mathrm{~N} .
\end{aligned}
$$


21. Computation of $D_{99}$ - uniaxial torsional rotations with $\chi_{z x}=1$ (Fig. 30). This results in

$$
\begin{aligned}
U_{\mathrm{FEM}} & =1229.97 \mathrm{~mJ}, \\
D_{99} & =2 U_{\mathrm{FEM}} / V=156.03 \mathrm{~N} .
\end{aligned}
$$

\section{Conclusions}

Using the linear homogenization technique similar to developed in [40], we have obtained full matrices of elastic moduli which relate the stress and couple stress tensors with the tensors of strain and microcurvature. Here, we have considered a metal foam of porosity $93.66 \%$. We have shown that this foam can be treated as material couple stress. In a certain sense, the presented results solved a problem of reconstruction of constitutive equations for a given microstructured material, which was treated as one of principal problem of continuum mechanics $[14,15]$. In fact, in addition to other material data these matrices give a source for further modeling of the considered foam and other porous materials in the framework of the couple stress theory, whereas the developed technique can be applied for other materials with complex microstructure. In particular, such beam-lattice structures could be interesting also for non-mechanical applications considering electroelastic coupling, see, e.g., [50,51].

Acknowledgements Authors would like to thank Professors R. Pęcherski and Z. Nowak from the Institute of Fundamental Technological Research Polish Academy of Science for shearing geometrical model of the foam obtained from microtomography scans.

Open Access This article is licensed under a Creative Commons Attribution 4.0 International License, which permits use, sharing, adaptation, distribution and reproduction in any medium or format, as long as you give appropriate credit to the original author(s) and the source, provide a link to the Creative Commons licence, and indicate if changes were made. The images or other third party material in this article are included in the article's Creative Commons licence, unless indicated otherwise in a credit line to the material. If material is not included in the article's Creative Commons licence and your intended use is not permitted by statutory regulation or exceeds the permitted use, you will need to obtain permission directly from the copyright holder. To view a copy of this licence, visit http://creativecommons.org/licenses/by/4.0/.

\section{References}

1. Ashby, M.F., Evans, A.G., Fleck, N.A., Gibson, L.J., Hutchinson, J.W., Wadley, H.N.G.: Metal Foams: A Design Guide. Butterworth-Heinemann, Boston (2000)

2. Gibson, L.J., Ashby, M.F.: Cellular Solids: Structure and Properties. Cambridge Solid State Science Series, 2nd edn. Cambridge University Press, Cambridge (1997)

3. Banhart, J.: Manufacturing routes for metallic foams. J. Miner. 52(12), 22-27 (2000)

4. Banhart, J., Ashby, M.F., Fleck, N.A. (eds.): Metal Foams and Porous Metal Structures. Verlag MIT Publishing, Bremen (1999)

5. Altenbach, H., Öchsner, A. (eds.): Cellular and Porous Materials in Structures and Processes. CISM Courses and Lectures, vol. 521. Springer, Wien (2011)

6. Lakes, R.S.: Foam structures with a negative Poisson's ratio. Science 235, 1038-1040 (1987)

7. Lim, T.-C.: Auxetic Materials and Structures, Engineering Materials. Springer, Singapore (2015)

8. Lakes, R.S.: Experimental microelasticity of two porous solids. Int. J. Solids Struct. 22(1), 55-63 (1986)

9. Rueger, Z., Lakes, R.S.: Experimental Cosserat elasticity in open-cell polymer foam. Philos. Mag. 96(2), 93-111 (2016)

10. Rueger, Z., Lakes, R.S.: Cosserat elasticity of negative Poisson's ratio foam: experiment. Smart Mater. Struct. 25(5), 054004 (2016)

11. Rueger, Z., Lakes, R.S.: Strong Cosserat elasticity in a transversely isotropic polymer lattice. Phys. Rev. Lett. 120(6), 065501 (2018)

12. Rueger, Z., Lakes, R.S.: Experimental study of elastic constants of a dense foam with weak Cosserat coupling. J. Elast. 137, 101-115 (2019)

13. Rueger, Z., Ha, C.S., Lakes, R.S.: Cosserat elastic lattices. Meccanica 54, 1983-1999 (2019). https://doi.org/10.1007/s11012019-00968-7

14. Truesdell, C.: A First Course in Rational Continuum Mechanics. Academic Press, New York (1977)

15. Truesdell, C., Noll, W.: The Non-linear Field Theories of Mechanics, 3rd edn. Springer, Berlin (2004)

16. Diebels, S., Steeb, H.: Stress and couple stress in foams. Comput. Mater. Sci. 28(3-4), 714-722 (2003)

17. Ehlers, W., Ramm, E., Diebels, S., d'Addetta, G.D.A.: From particle ensembles to Cosserat continua: homogenization of contact forces towards stresses and couple stresses. Int. J. Solids Struct. 40(24), 6681-6702 (2003)

18. Rahali, Y., Giorgio, I., Ganghoffer, J.F., dell'Isola, F.: Homogenization à la Piola produces second gradient continuum models for linear pantographic lattices. Int. J. Eng. Sci. 97, 148-172 (2015)

19. Boutin, C., dell'Isola, F., Giorgio, I., Placidi, L.: Linear pantographic sheets: asymptotic micro-macro models identification. Math. Mech. Complex Syst. 5(2), 127-162 (2017) 
20. Turco, E., dell'Isola, F., Cazzani, A., Rizzi, N.L.: Hencky-type discrete model for pantographic structures: numerical comparison with second gradient continuum models. Z. Angew. Math. Phys. 67(4), 85 (2016)

21. dell'Isola, F., Giorgio, I., Pawlikowski, M., Rizzi, N.: Large deformations of planar extensible beams and pantographic lattices: heuristic homogenisation, experimental and numerical examples of equilibrium. Proc. R. Soc. Lond. A 472(2185), 20150790 (2016)

22. dell'Isola, F., Seppecher, P., Spagnuolo, M., Barchiesi, E., Hild, F., Lekszycki, T., Giorgio, I., Placidi, L., Andreaus, U., Cuomo, M., et al.: Advances in pantographic structures: design, manufacturing, models, experiments and image analyses. Contin. Mech. Thermodyn. 31, 1231-1282 (2019)

23. Abdoul-Anziz, H., Seppecher, P.: Strain gradient and generalized continua obtained by homogenizing frame lattices. Math. Mech. Complex Syst. 6(3), 213-250 (2018)

24. Khakalo, S., Balobanov, V., Niiranen, J.: Modelling size-dependent bending, buckling and vibrations of 2D triangular lattices by strain gradient elasticity models: applications to sandwich beams and auxetics. Int. J. Eng. Sci. 127, 33-52 (2018)

25. Eremeyev, V.A.: Two- and three-dimensional elastic networks with rigid junctions: modelling within the theory of micropolar shells and solids. Acta Mech. 230(11), 3875-3887 (2019). https://doi.org/10.1007/s00707-019-02527-3

26. Rahali, Y., Ganghoffer, J.-F., Chaouachi, F., Zghal, A.: Strain gradient continuum models for linear pantographic structures: a classification based on material symmetries. J. Geom. Symmetry Phys. 40, 35-52 (2015)

27. Rahali, Y., Dos Reis, F., Ganghoffer, J.-F.: Multiscale homogenization schemes for the construction of second-order grade anisotropic continuum media of architectured materials. Int. J. Multiscale Comput. Eng. 15(1), 1-44 (2017)

28. Rahali, Y., Eremeyev, V.A., Ganghoffer, J.-F.: Surface effects of network materials based on strain gradient homogenized media. Math. Mech. Solids 25(2), 389-406 (2020). https://doi.org/10.1177/1081286519877684

29. Nowacki, W.: Theory of Asymmetric Elasticity. Pergamon-Press, Oxford (1986)

30. Eringen, A.C.: Microcontinuum Field Theory. I. Foundations and Solids. Springer, New York (1999)

31. Toupin, R.A.: Elastic materials with couple-stresses. Arch. Ration. Mech. Anal. 11(1), 385-414 (1962)

32. Mindlin, R.D.: Micro-structure in linear elasticity. Arch. Ration. Mech. Anal. 16(1), 51-78 (1964)

33. Mindlin, R.D., Eshel, N.N.: On first strain-gradient theories in linear elasticity. Int. J. Solids Struct. 4(1), 109-124 (1968)

34. dell'Isola, F., Sciarra, G., Vidoli, S.: Generalized Hooke's law for isotropic second gradient materials. R. Soc. Lond. Proc. Ser. A 465(2107), 2177-2196 (2009)

35. Auffray, N., Le Quang, H., He, Q.-C.: Matrix representations for 3D strain-gradient elasticity. J. Mech. Phys. Solids 61(5), 1202-1223 (2013)

36. Auffray, N., Dirrenberger, J., Rosi, G.: A complete description of bi-dimensional anisotropic strain-gradient elasticity. Int. J. Solids Struct. 69, 195-206 (2015)

37. Auffray, N., Kolev, B., Olive, M.: Handbook of bi-dimensional tensors: part I: harmonic decomposition and symmetry classes. Math. Mech. Solids 22(9), 1847-1865 (2017)

38. Bertram, A.: Compendium on Gradient Materials Including Solids and Fluids, 4th edn. TU Berlin, Berlin (2019)

39. Yang, F.A.C.M., Chong, A.C.M., Lam, D.C.C., Tong, P.: Couple stress based strain gradient theory for elasticity. Int. J. Solids Struct. 39(10), 2731-2743 (2002)

40. Goda, I., Rahouadj, R., Ganghoffer, J.-F., Kerdjoudj, H., Siad, L.: 3D couple-stress moduli of porous polymeric biomaterials using $\mu \mathrm{CT}$ image stack and FE characterization. Int. J. Eng. Sci. 100, 25-44 (2016)

41. Veyhl, C., Belova, I.V., Murch, G.E., Öchsner, A., Fiedler, T.: Thermal analysis of aluminium foam based on micro-computed tomography. Materialwiss. Werkstofftech. 42(5), 350-355 (2011)

42. Vesenjak, M., Krstulović-Opara, L., Ren, Z., Öchsner, A., Domazet, Ž.: Experimental study of open-cell cellular structures with elastic filler material. Exp. Mech. 49(4), 501 (2009)

43. Hossein Hosseini, S.M., Öchsner, A., Fiedler, T.: Numerical prediction of the effective thermal conductivity of open-and closed-cell foam structures. In: Defect and Diffusion Forum, vol. 297, pp. 1210-1217. Trans Tech Publishing (2010)

44. Fiedler, T., Öchsner, A., Gracio, J., Kuhn, G.: Structural modeling of the mechanical behavior of periodic cellular solids: open-cell structures. Mech. Compos. Mater. 41(3), 277-290 (2005)

45. Pęcherski, R .B., Nowak, M., Nowak, Z.: Virtual metallic foams. Application for dynamic crushing analysis. Int. J. Multiscale Comput. Eng. 15(5), 431-442 (2017)

46. Hill, R.: The essential structure of constitutive laws for metal composites and polycrystals. J. Mech. Phys. Solids 15(2), 79-95 (1967)

47. Qu, J., Cherkaoui, M.: Fundamentals of Micromechanics of Solids. Wiley, Hoboken (2006)

48. Eremeyev, V.A., Pietraszkiewicz, W.: Material symmetry group of the non-linear polar-elastic continuum. Int. J. Solids Struct. 49(14), 1993-2005 (2012)

49. Eremeyev, V.A., Pietraszkiewicz, W.: Material symmetry group and constitutive equations of micropolar anisotropic elastic solids. Math. Mech. Solids 21(2), 210-221 (2016)

50. Sharma, N.D., Maranganti, R., Sharma, P.: On the possibility of piezoelectric nanocomposites without using piezoelectric materials. J. Mech. Phys. Solids 55(11), 2328-2350 (2007)

51. Eremeyev, V.A., Ganghoffer, J.-F., Konopińska-Zmysłowska, V., Uglov, N.S.: Flexoelectricity and apparent piezoelectricity of a pantographic micro-bar. Int. J. Eng. Sci. 149, 103213 (2020)

Publisher's Note Springer Nature remains neutral with regard to jurisdictional claims in published maps and institutional affiliations. 